\title{
LA POLÍTICA DE DESARROLLO RURAL INTEGRADO EN LA UNIÓN EUROPEA: VIEJOS ENFOQUES Y NUEVAS TENDENCIAS
}

\author{
POR \\ ASCENSIÓN CALATRAVA ANDRÉS \\ y \\ ANA MELERO GUILLÓN
}

Introducción: el mundo rural en mutación

El mundo rural en la Comunidad, al igual que ocurre en otros países industrializados, está sufriendo cambios notables cuyas grandes tendencias pueden concretarse en las siguientes:

- Profunda reestructuración del sector agrario con una disminución importante de la superficie destinada a uso agrícola ${ }^{1}$, fuerte reducción de la mano de obra ${ }^{2}$, modernización e intensificación de

${ }^{1}$ Entre 1961-1965 y 1983 la superficie agraria utilizada se ha reducido en un 8\% (11 millones de Ha.) lo que no implica que no haya aumentado la superficie dedicada a los cultivos con precios de intervención (cereales, trigo blando, remolacha azucarera, etc.).

2 Durante el período 1958-1973 en la Europa de los Seis había un agricultor por minuto que abandonaba su actividad, esta tendencia ha ido continuando y así si en 1973 el empleo agrícola respecto del empleo total significaba el 11,3\%, en 1991 la proporción había descendido al 6,1\%. En España la proporción ha variado del 23,6\% al 10,4\%.

Ascensión Calatrava Andrés y Ana Melero Guilló. Instituto de Economía y Geografía (C.S.I.C.). Madrid.

Estudios Geográficos

Tomo LX, n. ${ }^{\circ} 237$, octubre-diciembre 1999 
los procesos de producción y tendencias a la polarización estructural ${ }^{3}$.

- Mayor diversificación económica originada por la pérdida de la importancia relativa del valor añadido por la agricultura en el PIB total $^{4}$; por la aparición de nuevas actividades; por el mejoramiento de las industrias locales a través de la innovación (OCDE 1992; Canto, C. del y Casabianca, F. de 1996).

- Nacimiento de empresas rurales, financiadas bien con fondos personales, o con fondos de un pequeño grupo de personas asociadas.

- Indicios de inversión de las tendencias de despoblación en ciertas regiones rurales de la UE, después del éxodo rural generalizado de los años sesenta y vinculado a las migraciones interregionales e internacionales ${ }^{5}$, situación que está originando la creación de centros urbanos menores (subpolos de actividad económica) y que no implica ni la vuelta masiva a las zonas rurales, ni que esta tendencia se esté produciendo de forma homogénea (Calatrava, A., 1994).

- Protección del medio ambiente rural, dado que el crecimiento a largo plazo sólo puede garantizarse en asociación con la naturaleza (Calatrava, A. y Melero, A., 1995).

El que los cambios iniciados se mantengan y se intensifiquen depende en gran medida de que exista fuerte interconexión entre los responsables de ese desarrollo: UE, Estados miembros, e interlocutores regionales y locales, aportando cada uno lo que sea de su competencia y atribuciones.

En la Agenda 2000 la Comisión Europea ha tomado en cuenta esas necesidades y se están dando los pasos necesarios para que en el futuro Europa sea más fuerte y más amplia, mediante la renovación y la re-

${ }^{3}$ Aumenta la parte de superficie agraria y producción correspondiente a explotaciones de más de $50 \mathrm{Ha}$, subsistiendo gran número de pequeñas explotaciones, bien a tiempo parcial o bien con un importante subempleo, representando estas últimas categorías 1/3 de las explotaciones comunitarias.

${ }^{4}$ En 1980, en la CEE-12, la parte del valor añadido neto al coste de los factores de la agricultura, en el PIB neto al coste de los factores, representaba el 3,6\% descendiendo al 2,2\% en 1993. En España las cifras han variado del 6,6\% al 3,5\%.

5 Aunque en términos absolutos la población rural sigue descendiendo, el medio rural muestra signos de recobrar una cierta vitalidad demográfica: el descenso poblacional se ha ralentizado al disminuir sensiblemente el éxodo rural, pero sigue existiendo crecimiento vegetativo negativo en las áreas rurales (García Sanz, B., 1996). 
forma de las políticas comunitarias y aplicando nuevos Reglamentos para la utilización de los Fondos estructurales.

En este trabajo, tras constatar las características diferenciales de las zonas rurales de los países de la UE, se aborda el tema de la evolución que ha experimentado la atención al desarrollo rural en la UE, desde la década de los 80 hasta nuestros días, las expectativas que se abren después del año 2000 y las perspectivas que se ofrecen para la participación de la población rural en su propio desarrollo, ya que los interlocutores locales, por su mayor imbricación con el territorio, tienen un papel destacado e ineludible en la difícil obra de lograr una mayor calidad de vida en las regiones y en los países, que pasa por una participación directa en las posibilidades de creación de empleo, utilizando los medios que se pongan a su alcance.

\section{La diversidad de las áreas rurales en la $U E$}

La inexistencia de una definición concreta de áreas rurales ha movido que los Estados miembros hayan desarrollado sus propias definiciones de áreas rurales para actuar a nivel de políticas nacionales basándose en criterios socio-económicos. Entre ellos, el más utilizado para definir la frontera entre lo rural y lo urbano es el de densidad de población, aunque no hay coincidencia de criterios entre países. Ante esta situación, la OCDE y la UE a través de EUROSTAT, han establecido criterios para describir las diferentes situaciones existentes en las zonas rurales de la $\mathrm{UE}^{6}$.

Atendiendo al criterio de la OCDE, se distinguen dos niveles jerárquicos de unidades territoriales:

${ }^{6}$ Los indicadores estadísticos constituyen una de las herramientas más utilizadas para definir áreas rurales, a pesarr de que cualquier definición estadística de lo rural plantea problemas metodológicos temibles (Berger, A. y Rouzier, J., 1995). Existen numerosos enfoques para conceptualizar la ruralidad al ser un espacio heterogéneo que puede ser cuestionado de diversas maneras (Blanc, M., 1997): el enfoque espacial que se basa en la teoría de los lugares centrales y los modelos del geógrafo W. Christaller y el economista A. Lösch; el enfoque territorial que focaliza la atención en la organización interna de las economías locales, el enfoque constructivista, que tiene en cuenta los grupos sociales. Últimamente, para definir y localizar espacialmente zonas rurales y llegar a establecer una tipología, están tomando auge las técnicas telemáticas que utilizan aplicaciones de redes neuronales (Blunden, J. R., et al., 1998).

$$
-581-
$$


Nivel de comunidad local (NUTS 5) ${ }^{7}$ : las áreas rurales son las que tienen una densidad de población menor de $150 \mathrm{hab} . / \mathrm{km}^{2}$.

Nivel regional (principalmente NUTS 3): considera tres tipos de regiones dependiendo de la población que en ella vive: Regiones predominantemente rurales: alrededor del 50\% de la población vive en comunidades rurales.-Regiones significativamente rurales: entre el 15 y el $50 \%$ de la población vive en comunidades rurales.-Regiones predominantemente urbanas: menos del $15 \%$ de la poblacion vive en comunidades rurales.

El enfoque de EUROSTAT se basa en el grado de urbanización, clasificándose las regiones de Europa dentro de tres clases:

Zonas densamente pobladas: son los grupos de municipios contiguos con densidad de población individual superior a $500 \mathrm{hab} / \mathrm{km}^{2}$, y en zonas con una población total de al menos 50.000 habitantes.-Zonas intermedias: cuando con la población total del grupo anterior, cada grupo de municipios tiene más de $100 \mathrm{hab} / \mathrm{km}^{2}$.—Zonas escasamente poblada: las restantes.

La DG VI, en su documento de trabajo «PAC 2000, Situación y Perspectivas: Desarrollos rurales», aparecido en julio de 1997, ajusta el umbral de densidad de población en $100 \mathrm{hab} / \mathrm{km}^{2}$. De acuerdo con este parámetro, cerca del 17,5\% de la población de la UE vive en comunidades rurales (cubriendo el 81\% del territorio de la UE) (Cuadro I). A nivel de países, las diferencias son notables, yendo desde menos del $5 \%$ en Holanda y Bélgica hasta superar el 50\% en Finlandia y Suecia. En España, la población que vive en comunidades rurales es casi el $25 \%$ de la población total (segunda columna del mencionado Cuadro I).

${ }^{7}$ La clasificación NUTS (Nomenclatura de Unidades Territoriales Estadísticas), agrupa datos regionales y contiene varios niveles:

- Nivel 0: Son los estados miembros (15 unidades).

- Nivel 1: Comprende 77 regiones (con diferentes denominaciones nacionales, así son las Régions en Bélgica y los Länder en Alemania).

- Nivel 2: Comprende 206 regiones (equivalen, por ejemplo, a las Regioni de Italia y a las Comunidades Autónomas de España).

- Nivel 3: Incluye 1.031 regiones (Départements de Francia y Länd de Suecia).

En los últimos años, la creciente demanda en cuanto a información de carácter local (infra-regional) llevó a poner en marcha en 1990 el proyecto SIRE (Sistema Europeo de Información Intra-regional) en el que se han definido dos niveles adicionales de la NUTS, el 4 y el 5. No obstante, sólo el nivel 5, el más preciso, ha sido perfilado en todos los estados miembros y corresponde a los municipios.

$$
-582-
$$


CuAdro I

POBLACIÓN RURAL EN LOS PAÍSES DE LA UE 15

\begin{tabular}{lcccc}
\hline & Población en & \multicolumn{2}{c}{ Población por tipo de regiones } \\
\cline { 3 - 5 } Países & $\begin{array}{c}\text { comunidades } \\
\text { rurales }\end{array}$ & $\begin{array}{c}\text { Predominan- } \\
\text { temente } \\
\text { Rural }\end{array}$ & $\begin{array}{c}\text { Significati- } \\
\text { vamente } \\
\text { Rural }\end{array}$ & $\begin{array}{c}\text { Predominan- } \\
\text { temente } \\
\text { Urbanizada }\end{array}$ \\
\cline { 3 - 5 } & & \multicolumn{2}{c}{ (\% de la población nacional) } \\
\hline Suecia & 66,8 & 63,2 & 17,7 & 19,1 \\
Finlandia & 50,6 & 58,8 & 41,1 & 0,0 \\
Irlanda & 43,1 & 46,6 & 15,1 & 38,3 \\
Austria & 34,6 & 30,2 & 28,9 & 41,0 \\
Dinamarca & 32,4 & 39,6 & 31,3 & 29,1 \\
Grecia & 30,8 & 28,1 & 28,3 & 43,6 \\
España & 24,4 & 12,7 & 41,5 & 45,8 \\
Francia & 23,7 & 10,5 & 56,5 & 32,9 \\
Portugal & 21,2 & 18,1 & 22,8 & 59,1 \\
Luxemburgo & 19,3 & $n . d$. & 100,0 & n.d. \\
Italia & 14,1 & 4,1 & 27,1 & 68,6 \\
Alemania & 12,0 & 5,4 & 25,2 & 69,3 \\
Reino Unido & 8,7 & 1,0 & 18,7 & 80,3 \\
Bélgica & 4,9 & 3,4 & 4,9 & 91,7 \\
Holanda & 3,1 & 0,0 & 6,7 & 93,3 \\
\hline UE-15 & 17,5 & 9,7 & 29,8 & 60,5 \\
\hline Superf. UE-15 & $80,9 \%$ & $47,00 \%$ & $37,40 \%$ & $15,60 \%$ \\
\hline & & & &
\end{tabular}

* Población de comunidades locales con densidad de población inferior a $100 \mathrm{hab} / \mathrm{km}^{2}$.

Fuente: Elaboración propia con datos de la Comisión Europea. Dirección General para la Agricultura (DG VI). 1997.

Utilizando la metodología de la OCDE se deduce que cerca del 10\% de la población de la UE vive y trabaja en zonas predominantemente rurales, muchas de ellas remotas, y cubriendo el $47 \%$ de la superficie de la Unión, casi el 30\% puebla zonas significativamente rurales (37,4\% de la superficie total) y el $60 \%$ de la población habita en zonas predominantemente urbanizadas (menos del 16\% del área total de la UE) (columnas 3,4 , y 5 del citado Cuadro I).

$$
-583-
$$


La atención al desarrollo rural en el contexto de las políticas comunitarias

La arquitectura de la actual política estructural de desarrollo rural es básicamente el resultado de la superposición de dos políticas paralelas: la Política Agrícola y Rural, nacida del Tratado de Roma y la Política Regional de la $\mathrm{CE}$, creada a mediados de los setenta y apoyada en el FEDER. A esas dos políticas, hay que añadir la Política de Pesca y la Política Medioambiental.

Las respuestas dadas por la Comunidad Europea al desarrollo rural desde la década de los ochenta se contemplaron dentro de la PAC (con la excepción de las acciones dirigidas a la agricultura de montaña y a determinadas zonas desfavorecidas) y se abordaron desde una óptica de mejora de la competitividad de las explotaciones agrarias y de apoyo a la renta de los agricultores. También desde los ochenta, y debido a la crisis económica europea y en las sucesivas ampliaciones de la CEE, la Comisión Europea se ha visto obligada a: concentrar sus intervenciones estructurales, sobre todo en las regiones subdesarrolladas; introducir la cofinanciación y los programas multianuales por la Comisión y los países miembros, y financiar acciones para promover el desarrollo endógeno potencial de algunas regiones.

La nueva estrategia para el desarrollo rural se empezó a plantear en el llamado «Libro Verde» ${ }^{8}$ de la Comisión de 1985 (COM (85) 333), y posteriormente en el Informe de la Comisión «El Futuro del Mundo Rural», aparecido en 1988, se tomaron los problemas rurales en seria consideración empezando a hacerles frente apuntando soluciones y medidas (ver Cuadro II) que tomaron cuerpo en la reforma de 1988 de los Fondos estructurales, donde se hablaba del Desarrollo Rural Integrado. Y más adelante, en 1993, las zonas rurales recibían reconocimiento político en el seno de la UE mediante una cita específica en el Artículo 130 A del Tratado de Maastricht, identificándolas como una prioridad para la asistencia bajo la política de cohesión económica y social ${ }^{9}$.

8 El Libro Verde subrayaba por un lado, la necesidad de integrar el desarrollo agrario dentro de un enfoque multisectorial de diversificación económica de las zonas rurales. Y por otro, ponía de manifiesto el papel clave de la agricultura, tanto en sus funciones estratégicas, económicas y sociales, como en relación con la protección del medio ambiente y la conservación del patrimonio y los recursos naturales.

9 «A fin de promover un desarrollo armonioso del conjunto de la Comunidad, ésta desarrollará y proseguirá su acción encaminada a reforzar su cohesión económica y social. 
CUADRO II

EL MUNDO RURAL FRENTE A LAS PRESIONES DEL MUNDO MODERNO: PROBLEMAS Y MEDIDAS PARA SU SOLUCIÓN

\begin{tabular}{lcc}
\multicolumn{1}{c}{ Problemas } & Soluciones & Medidas \\
\hline \multicolumn{2}{c}{ Agricultura } \\
$\begin{array}{l}\text { Explotación excesiva } \\
\text { del suelo }\end{array}$ & $\begin{array}{c}\text { Ordenación del terri- } \\
\text { torio }\end{array}$ & $\begin{array}{c}\text { Delimitación de zonas agrarias (planes de } \\
\text { ocupación del suelo) } \\
\text { Contaminación }\end{array}$ \\
& $\begin{array}{c}\text { Protección del medio } \\
\text { ambiente }\end{array}$ & $\begin{array}{c}\text { Concentración parcelaria; mantenimento } \\
\text { de una agricultura competitiva, medidas }\end{array}$ \\
& & que favorezcan la adaptación a cultivos \\
que requieren menos productos químicos & Extensificación
\end{tabular}

\section{Bosques}

Frecuentación exce- Predominio de la fun- Protección del bosque (limitación de la dessiva ción social (asueto, forestación, etc.)

Contaminación descanso, ocio) Repoblación forestal con intenciones merespetando los as- dioambientales, recreativas y paisajístipectos medioam- cas

bientales Creación de espacios forestales equilibrados en relación con las zonas urbanas

\begin{tabular}{|c|c|c|}
\hline \multicolumn{3}{|c|}{ Medio ambiente } \\
\hline $\begin{array}{l}\text { Presión periurbana } \\
\text { (deterioro de espa- } \\
\text { cios verdes) } \\
\text { Agricultura intensiva } \\
\text { (contaminación de- } \\
\text { bida a abonos, pla- } \\
\text { guicidas, destruc- } \\
\text { ción de biotopos) }\end{array}$ & $\begin{array}{l}\text { Utilización racional } \\
\text { del espacio } \\
\text { Reglamentación (nor- } \\
\text { mas) } \\
\text { Incentivos (modifica- } \\
\text { ciones de las técni- } \\
\text { cas de cultivo) }\end{array}$ & $\begin{array}{l}\text { Planes de ocupación del suelo } \\
\text { Estudios de impacto medioambiental } \\
\text { Mantenimiento de zonas agrarias y fores- } \\
\text { tales } \\
\text { Normas de calidad del aire, agua, etc. } \\
\text { Reglamentación sobre sustancias peligrosas } \\
\text { Servicios de divulgación; desarrollo de téc- } \\
\text { nicas no contaminantes } \\
\text { Designación y conservación efectiva de zo- } \\
\text { nas de protección }\end{array}$ \\
\hline
\end{tabular}

\section{Servicios}

Competencia/atrac- Conexión con la red ción de núcleos urbanos

Calidad de vida ligada a los inconvenientes de las estructuras urbanas periféricas (ciudades domitorios, etc.)
Política de innovación y calidad frente a la competencia de los núcleos urbanos

Desarrollo de nuevas actividades terciarias de servicios
Creación de centros de servicios (oficinas) o disponibilidad de servicios próximos tanto a personas como a empresas

Mejora de las infraestructuras de comunicación

Creación de tarifas preferenciales para los servicios públicos (telecomunicaciones, transporte, fiscalidad, etc.)

Desarrollo del turismo rural (infraestructuras de alojamiento y esparcimiento; organización y revalorización de las prestaciones turísticas existentes) 


\begin{tabular}{|c|c|c|}
\hline \multicolumn{3}{|c|}{ CUADRO II (continución) } \\
\hline \multicolumn{3}{|c|}{ Actividades secundarias } \\
\hline $\begin{array}{l}\text { Congestión urbana, } \\
\text { falta de actividades } \\
\text { alternativas, vacío } \\
\text { en torno a las ciu- } \\
\text { dades (sur de la } \\
\text { Comunidad) }\end{array}$ & $\begin{array}{l}\text { Creación de nuevos } \\
\text { «polos» de desa- } \\
\text { rrollo económico } \\
\text { que descongestio- } \\
\text { nen las aglomera- } \\
\text { ciones urbanas }\end{array}$ & $\begin{array}{l}\text { Desarrollo de un modelo «parque indus- } \\
\text { trial y tecnológico» } \\
\text { Apoyo a las iniciativas locales fuera de los } \\
\text { grandes centros. }\end{array}$ \\
\hline
\end{tabular}

Fuente de datos: Elaboración propia a partir de El futuro del mundo rural (COM (88) 501 final). Boletín de las Comunidades Europeas. Suplemento 4/88.

El reconocimiento de las necesidades específicas de la áreas rurales identificado en 1988 y reforzado en 1993, supuso el «nacimiento» de una Política específica de desarrollo rural con objetivos definidos, instrumentos y medios financieros.

Para la Política de desarrollo rural se han identificado cuatro principales objetivos: Promover la cohesión económica y social, mediante el mantenimiento y la creación de empleos.-Superar las barreras al desarrollo estimulando la diversificación, mejorando las infraestructuras y facilitando el acceso a las nuevas tecnologías. - Aumentar la calidad de vida (conservando el medio ambiente, dando acceso a los servicios básicos...).--Mantener comunidades rurales viables preservando sus tradiciones y su cultura.

Las medidas que contribuyen al desarrollo rural, llegan por varios caminos: Los Programas de los Fondos estructurales ayudan al ajuste estructural por dos vías: a través de la asistencia a las regiones mediante medidas horizontales, y con la iniciativa LEADER.-Las medidas de acompañamiento de la PAC, que tienen por fin ayudar a las comunidades agrarias a adaptarse a las consecuencias de los cambios en los regímenes de mercados y proporcionan una nueva fuente de ingresos.-Otras medidas derivadas de la Política de Pesca y de la de Política Medioambiental, las cuales disponen de instrumentos financieros que se aplican en determinadas áreas rurales.

La Comunidad propondrá en particular reducir las diferencias entre los niveles de desarrollo de las diversas regiones y el retraso de las regiones menos favorecidas, incluidas las zonas rurales» (art. $130 \mathrm{~A}$ del Tratado CE).

$$
-586-
$$


Medidas estructurales de la UE: el impulso al desarrollo a través de la reforma de los fondos estructurales

Entre 1985 y 1994, y por motivos concretos, se han ido creando los instrumentos estructurales que proporcionan fondos para el desarrollo de las regiones de la UE. Estos fondos han experimentado dos reformas en su historia: la primera, realizada en 1988 con motivo del nuevo marco que originó la entrada en vigor en 1987 del Acta Única que obligó a una revisión profunda de las normas de funcionamiento de los Fondos estructurales entonces existentes: FEDER, FSE, y FEOGA ${ }^{10}$.

La segunda reforma se inició en 1993. La entrada en vigor del Tratado de la UE obligó a una revisión de los reglamentos que regían el funcionamiento y la aplicación de los Fondos estructurales y se creó un nuevo instrumento financiero, el IFOP, que se unía a los Fondos estructurales existentes, con el fin de prestar ayuda a las regiones de la pesca ${ }^{11}$. La ampliación de la UE con la entrada de Austria, Suecia y Finlandia y la existencia en los dos últimos países de regiones necesitadas de ayuda para su desarrollo debido a la escasa densidad poblacional, motivó la creación del Objetivo 6, que conjuntamete con los Objetivos 1 y 5 son los más relevantes para promover la diversificacion de las zonas rurales (Reglamento CEE núm 20183/93 que modifica el Reglamento núm. 4253/88).

Los objetivos de desarrollo prioritario que tiene a su cargo la política estructural y que se favorecen de diversos fondos financieros, tienen las siguientes metas a alcanzar (Comisión Europea, 1996):

OBJETIVO NÚM. 1: FOMENTAR EL DESARROLLO Y EL AJUSTE ESTRUCTURAL DE LAS REGIONES MENOS DESARROLLADAS

Meta a alcanzar: El Objetivo núm. 1, que abarca el 26,6\% de la población actual de la Unión, se dirige al ajuste estructural de las regiones menos desarro-

${ }^{10}$ A partir de esta reforma, la política estructural se articularía en torno a tres ejes principales: mayor selectividad, incremento de los medios y mejora de los métodos de intervención, concentrándose las actuaciones en un número limitado de objetivos, que hasta 1994 fueron los siguientes objetivos: 1) Desarrollo y ajuste estructural en las regiones menos desarrolladas.-2) Reconversión económica de zonas industriales en decadencia.3) Lucha contra el desempleo de larga duración, inserción profesional de jóvenes e integración de personas amenazadas de exclusión del mercado laboral).-4) Adaptación de los trabajadores a la evolución de los sistemas de producción industrial.-5a) Adaptación de las estructuras agrarias.-5b) Desarrollo y reajuste estructural de zonas rurales.

Los objetivos 1,2 y $5 \mathrm{~b}$ están limitados geográficamente a regiones subvencionables. Los objetivos 3,4 y $5 \mathrm{a}$, abarcan todo el territorio comunitario.

${ }_{11}$ El instrumento financiero de orientación a la pesca (IFOP) se creó por el Reglamento (CEE), núm. 2080/93 el 20 de julio de 1993. 
lladas, entre las cuales la mayoría cuenta con un producto interior bruto (PIB) per cápita inferior al $75 \%$ de la media comunitaria. La gran mayoría de ellas se caracterizan por un marcado grado de ruralismo, ingente proporción de activos agrícolas y densidad de población a menudo escasa así como por una situación periférica en el contexto europeo. Los esfuerzos globales de desarrollo llevados a cabo en estas regiones movilizan la mayor parte (70\%) de los recursos de los Fondos estructurales.

Fondos involucrados: FEDER, FSE, FEOGA-Orientacion, IFOP.

OBJETIVO NÚM. 2: RECONVERTIR LAS REGIONES, REGIONES FRONTERIZAS O PARTE DE REGIONES GRAVEMENTE AFECTADAS POR EL DECLIVE INDUSTRIAL

Meta a alcanzar: Reconversión de las zonas industriales en declive, para que se reintegren plenamente en el proceso de desarrollo económico. Estas zonas se han definido en función de su grado de industrialización, de la disminución de empleo industrial y del porcentaje de parados.

Fondos involucrados: FSE.

OBJETIVO NÚM. 3: COMBATIR EL PARO DE LARGA DURACIÓN Y FACILITAR LA INSERCIÓN PROFESIONAL DE LOS JÓVENES Y DE LAS PERSONAS EXPUESTAS A LA EXCLUSIÓN DEL MERCADO LABORAL

Meta a alcanzar: Lucha contra el paro prolongado, que requiere intervenciones en toda la Comunidad.

Fondos involucrados: FSE.

OBJETIVO NÚM 4: FACILITAR LA ADAPTACIÓN DE LOS TRABAJADORES Y TRABAJADORAS A LAS MUTACIONES INDUSTRIALES Y A LA EVOLUCIÓN DE LOS SISTEMAS PRODUCTIVOS

Meta a alcanzar: Inserción profesional de los jóvenes, que interesa igualmente a toda la Comunidad.

Fondos involucrados: FSE.

OBJETIVO NÚM. 5: FOMENTAR EL DESARROLLO RURAL:

a) Adaptando las estructuras agrarias en el marco de la reforma de la PAC: modernizando reestructurando la pesca.

b) Facilitando el desarrollo y el ajuste estructural de las zonas rurales.

Meta a alcanzar: Adaptación de las estructuras agrarias, en relación con la reforma general de la Política Agraria Común, y reestructuración del sector pes- 
quero (objetivo 5a) y el desarrollo de las zonas rurales desfavorecidas. El Objetivo núm. 5 b completa la asuncion de los territorios rurales con un $8,2 \%$ de la población, y aspira a la diversificación económica de las zonas rurales vulnerables. Se trata sobre todo de zonas de bajo nivel de desarrollo socioeconómico (calculado sobre la base del PIB per cápita) que responden a dos de los tres criterios principales: elevado índice de empleo agrícola, bajo nivel de renta agraria, y escasa densidad de población o tendencia marcada a la despoblación. Otros criterios tales como el aislamiento, la precariedad estructural del sector agrícola o las incidencias sobre el medio ambiente han sido tenidas en cuenta para la delimitación de las zonas elegibles.

No obstante, los recursos asignados al Objetivo núm. 5b mantienen un nivel cuantitativo relativamente modesto, si se comparan con las dotaciones financieras de los demás objetivos. Las acciones financiadas son susceptibles, sin embargo, de producir un destacado efecto multiplicador sobre el desarrollo del mundo rural.

Fondos involucrados: 5a. FEOGA-Orientación, IFOP.-5b. FEOGA-Orientación, FSE, FEDER.

\section{OBJETIVO NÚM. 6: PROPORCIONAR INSTRUMENTOS ESTRUCTURALES ESPECÍFICOS A ZONAS ESCASAMENTE POBLADAS}

(Objetivo creado a raíz de la última ampliación de la UE en enero de 1995, para las zonas de densidad de población inferior a $8 \mathrm{hab} . / \mathrm{km}^{2}$, y en las que las condiciones climáticas son especialmente rigurosas. Aproximadamente el 50\% de la superficie total de Suecia y el $60 \%$ de la de Finlandia entran dentro del Objetivo 6)

Meta a alcanzar: Prevenir el éxodo rural y mejorar las condiciones de vida en el campo; poner énfasis en aumentar el VA de la materia prima producida en la región transformándola en la propia región, y aplicar medidas que obtengan el máximo provecho de las posibilidades locales.

Fondos involucrados: FEOGA-Orientación.

Teniendo en cuenta esta información, los programas de desarrollo rural (PDR) que se llevan a cabo en esas regiones se benefician de los Fondos estructurales FEOGA-Orientación, FEDER, FSE e IFOP. Es importante hacer notar que las regiones incluidas en estos objetivos, representan conjuntamente cerca del 75\% del área de la UE 15 y casi el $35 \%$ de su población.

La Comisión de la Unión Europea, además de proporcionar recursos para el desarrollo de las regiones a través de los Fondos estructurales, también facilita financiación a través de los Nuevos Programas de Ini- 
ciativas Comunitarias (1994-99) y que son de aplicación en áreas diversas (Comisión Europea, 1994):

\section{CARÁCTER REGIONAL}

INTERREG II: A) Cooperación interregional (regiones fronterizas); B) Completar redes de energía para conectarlas a redes europeas más amplias (antigua iniciativa REGEN).

REGIS II: Desarrollo de las Regiones ultraperiféricas.

\section{DESARROLLO RURAL}

LEADER II: Desarrollo rural con enfoque local.

\section{EMPLEO Y DESARROLLO DE LOS RECURSOS HUMANOS}

Empleo-NOW: Fomentar la igualdad de oportunidades de empleo para las mujeres.

Empleo-HORIZON: Facilitar el acceso al mercado laboral de los minusválidos y otros.

Empleo-YOUTHSTART: Favorecer la integración de los jóvenes en el mercado de trabajo, en especial, los que carecen de formación o cualificación suficiente.

\section{CAMBIO INDUSTRIAL}

ADAPT: Adaptación de los trabajadores a las transformaciones industriales. RECHAR II (1994-97): Reconversión económica de las zonas mineras del carbón.

RESIDER II: Reconversión económica de las cuencas siderúrgicas más afectadas.

KONVER (1993-97): Diversificación económica de las zonas que dependen del sector militar.

RETEX (1992-97): Ayudar a las regiones menos desarrolladas, a las afectadas por el declive industrial y a las zonas rurales desfavorecidas que dependen estrechamente del sector textil y de la confección.

Textil-Confección en Portugal: Fomentar la modernización de esos sectores en Portugal para facilitar su adaptación a la competencia internacional.

PYME: Ayudar a las pequeñas y medianas empresas, especialmente las de las regiones menos desarrolladas, a adaptarse al mercado único y a adquirir competitividad internacional. 


\section{POLÍTICA URBANA}

URBAN: Política urbana. Programas de desarrollo integrado de zonas urbanas.

\section{POLÍTICA PESQUERA}

PESCA: Reestructuración del sector pesquero.

Con independencia de la posible aplicación en las distintas zonas rurales de la mayoría de dichas iniciativas, la iniciativa LEADER (Liaison entre Activités de Développement de l'Economie Rurale) lanzada en 1991 (LEADER I) y renovada en 1994 para el período 1994-99 (LEADER II), es de específica aplicación para el desarrollo local.

Esta iniciativa se aplica en las zonas rurales de los Objetivos 1, 5b y 6, siendo sus beneficiarios los grupos de acción local (GAL), entendiendo como tales un conjunto de interlocutores públicos y privados que definen en común una estrategia y medidas innovadoras para el desarrollo de un territorio rural de dimensión local (menos de 100000 habitantes) y también, otros agentes colectivos o públicos del medio rural (colectivos locales, cámaras agrarias, de industria, de comercio, de artesanos, cooperativas, asociaciones, etc ), siempre y cuando su tema de actuación se sitúe dentro de una lógica de desarrollo de una zona rural ${ }^{12}$.

La aplicación de los fondos estructurales en las zonas rurales: los programas LEADER

El propósito de los programas LEADER es impulsar una línea integradora en las actividades de desarrollo de la economía rural dentro de

12 Esta iniciativa tiene como característica básica que todas las acciones deben incluirse en un plan de desarrollo integrado para la comunidad solicitante, que debe ser diseñado por la propia comunidad local. Con ello se asegura la complementariedad y produce un valor añadido a las acciones comprometidas.

Los objetivos específicos del LEADER II son cuatro: 1) Asegurar la continuidad de LEADER I con el apoyo a las iniciativas locales de desarrollo local ejemplares.-2) Dar apoyo a medidas innovadoras, demostrativas y transferibles que muestren las nuevas direcciones que puede tomar el desarrollo rural.-3) Aumentar los intercambios de experiencias y el intercambio de conocimientos (know-how) a través de una red europea de desarrollo rural.-4) Apoyar proyectos de cooperación transnacional que emanen de los agentes locales de las zonas rurales evocando su solidaridad.

$$
-591-
$$


un enfoque endógeno local con el fin de apoyar y estimular las iniciativas de los grupos de acción local. Según el texto de la directiva sobre los citados programas ${ }^{13}$, el primer objetivo marca la atención sobre lo siguiente: «La recuperación de un entramado socioeconómico suficientemente diversificado requiere un enfoque resueltamente endógeno y local que se apoye en la capacidad de acción y de los conocimientos técnicos. Por ello, con la iniciativa LEADER se pretende crear una red de grupos de acción local en favor de desarrollo rural que disponga de un margen de evaluación importante para aplicar localmente las actividades financiadas con las subvenciones globales nacionales. La actividad de cada grupo viene a añadirse a las medidas en favor del desarrollo local que se contemplan en los marcos comunitarios de apoyo» ${ }^{14}$ (Bandarra, N., 1992).

Paralelamente a este objetivo, otra característica importante de estos programas es tener vocación demostrativa y así servir de ejemplo para animar a otras acciones privadas en nuevas áreas, sirviendo a la vez de experiencia para posteriores intervenciones institucionales.

Con respecto a las acciones subvencionables por los programas LEADER, pueden referirse tanto a acciones relativas al desarrollo rural propiamente dicho (apoyo técnico del desarrollo rural; formación profesional y ayuda a la contratación; turismo rural; pequeña empresa, artesanía y servicios locales; valorización y comercialización «in situ» de la producción agraria, forestal y pesquera), como a acciones relativas a los grupos de desarrollo local (ayudas a la creación de grupos de desarrollo local, puesta en marcha, formación de animadores, etc; ayudas al equipamiento informático y telemático; asistencia técnica a los grupos; ayuda a la integración de los grupos en una red supranacional).

${ }^{13}$ El 19 de marzo de 1991 el Diario Oficial de las Comunidades Europeas publicaba la directiva de la Comisión sobre los programas LEADER (1991-93), suponiendo esta iniciativa la apuesta más importante comunitaria dentro de este ámbito. El 15 de junio de 1994 la Comisión de las Comunidades Europeas adoptó la iniciativa LEADER II (19941999).

${ }^{14}$ La selección de estos grupos de acción a veces de lleva a cabo conjuntamente por la Comisión y los Estados miembros teniendo en cuenta una serie de criterios tales como implantación local, solvencia, capacidad administrativa, calidad de los programas, conocimientos técnicos, experiencia y aceptación de las normas de funcionamiento de la red supranacional prevista en el artículo 6 de la directiva. 
Mecanismos de actuación

La aplicación de la política estructural al medio rural sigue generalmente tres fases:

Fase primera: elaboración de planes nacionales plurianuales por parte de los Estados miembros; Plan de Reconversión Regional y Social (PRD) para las regiones en declive (objetivo núm. 1) y Plan de desarrollo para Zonas Rurales (PDZR) para las zonas objetivo 5b. Lo más característico y novedoso de estos planes es el diseño de una estrategia de desarrollo regional previamente discutida con todas las Comunidades Autónomas y con la que se pretende potenciar el desarrollo socioeconómico de un área. En cuanto al esquema básico de trabajo a seguir para la elaboración de un Plan de Desarrollo Local, el Cuadro III recoge los puntos fundamentales, aspectos que están sugeridos en las normas impuestas por la Comisión de la CEE en el Programa LEADER.

\section{CuAdro III}

ESQUEMA BÁSICO DE UN PLAN O PROGRAMA DE DESARROLLO INTEGRADO CON ENFOQUE LOCAL

1. OBJETIVOS (Claros, concretos, sin solapes y poco numerosos)

1.1. Generales o globales

1.2. Específicos o sectoriales

1.3. Por ejes de desarrollo

2. ESTRATEGIA (Debe ser tan concreta que pueda expresarse mediante un lema)

3. SUBPROGRAMAS (No son imprescincibles). Se definen en número reducido para alcanzar cada objetivo y deben ser de menor rango y más concretos que éstos.

4. MEDIDAS. Se establecen para conseguir cada objetivo o subprograma en su caso. El grado de concreción ha de ser mayor aún. Suelen ser de carácter sectorial.

5. ACCIONES Y PROYECTOS. Las medidas se plasman en un paquete de acciones y proyectos concretos. Han de ser iniciativas o actividades de carácter económico, social, cultural, ecológico, etc., tan concretas que requieren localización, responsables que asuman el riesgo, determinación de presupuestos, fuentes financieras, estudio de viabilidad, etc. 
Elaborada su redacción, es importante someterla al consenso de las fuerzas implicadas y que el Plan vaya avalado tanto por la administración (regional y local) como por el resto de los agentes económicos locales (administraciones, patronales, asociaciones socio culturales y sindicatos).

Finalizado el diseño del conjunto de los planes de desarrollo local a nivel autonómico, las distintas Autonomías trasladarán dicha información a la Administración a fin de proceder a la fase de negociación financiera interna y a la aprobación correspondiente (Valcárcel-Resalt, G. 1993).

En cuanto a la metodología a seguir para la elaboración de un plan de desarrollo local de demanda institucional, el Cuadro IV refleja un esquema capaz de poder proporcionar las ideas y las claves necesarias que van a posibilitar el análisis de un posible desarrollo para un microterritorio determinado.

Fase II: elaboración de los Marcos Comunitarios de Apoyo (MCA). Para esta elaboración la Comisión, en base a los planes aprobados por los Estados miembros, negociará con el Comité de los entes regionales y locales ${ }^{15}$ con el fin de poder elaborar los Marcos Comunitarios de Apoyo para su posterior aprobación.

Una vez negociados y aprobados los MCA, continúa la Fase III: concesión de ayudas por parte de los distintos Estados miembros para la realización de los Planes. Estas demandas se canalizarán a través de programas operativos o solicitudes individuales para grandes proyectos esencialmente ${ }^{16}$ (Lázaro, L. 1995).

\section{Importancia financiera}

Durante el período 1989-1993 la importancia de las asignaciones económicas de la CEE de acuerdo con los objetivos programáticos as-

15 El número de representantes de los Estados miembros en el Comité de Entes Regionales y Locales es el siguiente: España, cinco representantes; Dinamarca, Bélgica, Portugal, Irlanda, Grecia y Países Bajos: dos representantes por país; Alemania, Reino Unido, Francia e Italia: seis representantes por país: Luxemburgo, 1 representante).

16 Sólo se admiten solicitudes para proyectos individuales cuando el coste total supera los 15 millones de Ecus (2.000 millones de pesetas aproximadamente) para inversiones en infraestructura, y los 10 millones de Ecus (aproximadamente 1.300 millones de pesetas) para inversiones productivas.

$$
-594-
$$


cendió a 59.164 millones de Ecus, dirigiéndose el $64,7 \%$ a las regiones Objetivo 1 y el 4,7\% a las regiones objetivo 5b, objetivos específicos para promover el desarrollo rural como quedo indicado anteriormente (Cuadro V).

\section{CUADRo IV}

ESQUEMA METODOLÓGICO PARA LA ELABORACIÓN DE UN PLAN DE DESARROLLO LOCAL

\section{PLANTEAMIENTO DE OBJETIVOS Y FILOSOFÍA}

2. RECOPILACIÓN DOCUMENTAL

Bibliográfica y demanda a redes internacionales, estudio de casos y visita de experiencias.

3. ESTUDIO DE LA NORMATIVA EN VIGOR

Análisis de la normativa en vigor. Incentivos oficiales.

Incidencia local del plan de desarrollo regional y de los programas operativos que le afecten.

4. ANÁLISIS Y EVALUACIÓN DE RECURSOS

Ponderación de las necesidades básicas de la población.

Identificación y evaluación de recursos.

Condicionantes, restricciones y estrangulamientos.

Potencialidades y puntos fuertes.

5. TRABAJOS DE CAMPO

Entrevistas en profundidad a líderes locales.

Entrevistas a testigos privilegiados.

Organización de grupos de discusión.

6. SELECCIÓN DE PROYECTOS

Preselección de acciones y proyectos concretos.

Ensayo de modelos de simulación dinámica: Análisis y Prospectiva.

Contrastación y ajustes por el método Delphi.

7. REDACCIÓN DEL PLAN Y NEGOCIACIÓN

Diseño del plan estratégico de desarrollo sostenible

Negociación y consenso con los responsables locales: «Comité de desarrollo» y «grupos de acción». 
Cuadro V

DESGLOSE DE LAS ASIGNACIONES POR OBJETIVOS (Millones de Ecus)

\begin{tabular}{lrrrr}
\hline & $\mathbf{1 9 8 9 - 1 9 9 3}$ & \% Total & $\mathbf{1 9 9 4 - 1 9 9 9}$ & \% Total \\
\hline Objetivo 1 & 38.300 & 64,73 & 93.810 & 74,33 \\
Objetivo 2 & 7.205 & 12,18 & 6.978 & 5,53 \\
Objetivos 3 y & 7.450 & 12,59 & 13.908 & 11,02 \\
Objetivo 5a & 3.415 & 5,77 & 5.369 & 4,26 \\
Objetivo 5b & 2.795 & 4,72 & 6.134 & 4,86 \\
\multicolumn{1}{c}{ TOTAL } & 59.164 & 100,00 & 126.188 & 100,00 \\
\hline
\end{tabular}

En cuanto a lo presupuestado para las regiones Objetivo 2, el porcentaje respecto del total alcanzó el 12,2\%, designándose conjuntamente a las regiones Objetivo 3 y 4 el $12,6 \%$ y el 5,7\% a las Objetivo 5 a.

Con respecto a lo proyectado para el período 1944-1999, la cuantía de los fondos estructurales asignados a los 12 Estados miembros se ha visto incrementada significativamente, de tal modo que las cantidades asignadas al Objetivo 1 (93.810 millones de Ecus) y las referidas al Objetivo $5 \mathrm{~b}$ (6.134 millones de Ecus) han supuesto incrementos positivos del $145 \%$ y del $119,5 \%$ respectivamente ${ }^{17}$.

Considerando la distribución de los fondos por regiones, el Gráfico 1 que recoge las asignaciones presupuestadas para los distintos Es-

17 A las cantidades asignadas a los 12 Estados miembros para el período 1994-99 hay que añadir las que en 1995 y cubriendo el período 1995-99 se destinaron para atender a las necesidades de desarrollo de los tres países que se adhirieron a la UE en 1995. La distribución de créditos de compromiso correspondientes al Objetivo 6 entre los nuevos Estados miembros consta en el Acta de Adhesión. La Comisión, una vez definidas las áreas subvencionables con arreglo a los objetivos 2 y 5b, estableció la distribución de las asignaciones previstas para los demás Objetivos. La distribución de las asignaciones de los Fondos estructurales entre 1995 y 1999 para los MCA y los DOCUP fue, en millones de Ecus, la siguiente:

\begin{tabular}{|c|c|c|c|c|c|}
\hline Estado & Objetivo $\mathbb{1}$ & Objetivo 2 & Objetivos 3,4 y $5 \mathbf{a}$ & Objetivo $\mathbf{5} \mathbf{b}$ & Objetivo 6 \\
\hline Austria & 165,6 & 101 & 783 & 411 & - \\
\hline Finlandia & - & 183 & 697 & 194 & 459,9 \\
\hline Suecia & - & 160 & 728 & 138 & 252,0 \\
\hline
\end{tabular}

(Comisión Europea, Fondos Estructurales y Fondo de Cohesión 1994-99. Textos reglamentarios y comentarios, Luxemburgo, OPOCE, 1996).

$$
-596-
$$




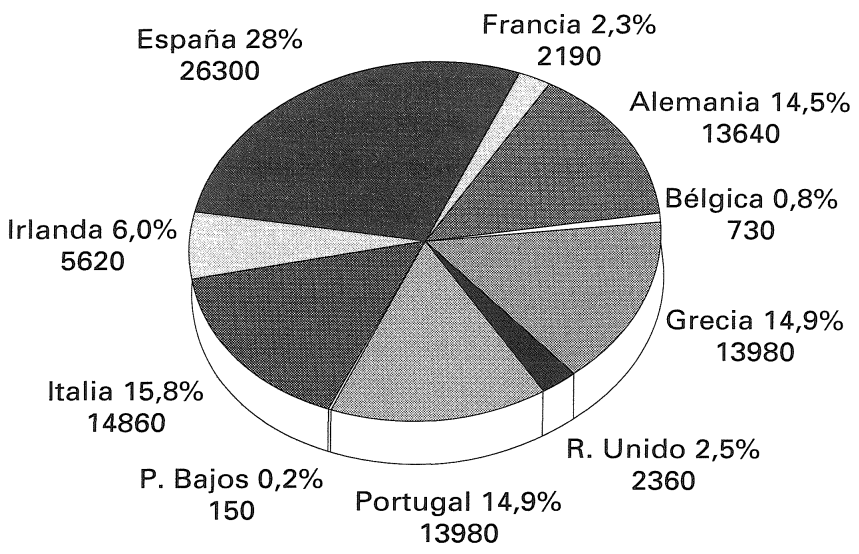

GRÁfICO 1.—Objetivo 1. 1994-1999 (mill. ecus)

Regiones con menor nivel de desarrollo.

Informe general CEE, año 1994.

tados europeos con respecto al Objetivo 1, muestra diferencias importantes en el reparto de los fondos, correspondiendo el porcentaje mayor a España con un $28 \%$ respecto del total, Italia sería el segundo país con un $15,8 \%$ y a Grecia y Alemania les correspondería el $3 .^{\circ}$ y $4 .^{\circ}$ lugar con porcentajes respectivos del 14,9 y 14,5\%; en cuanto al resto de los países europeos las cifras asignadas decrecen afectando de forma negativa en los porcentajes respectivos.

Analizando las asignaciones correspondientes al Objetivo 5b para el período 1994-1999, correspondería a Francia la mayor asignación con un $36,49 \%$ del total de los fondos asignados por este concepto, el $2 .^{\circ}$ lugar en el ranking lo ocuparía Alemania y en el tercer y cuarto lugar estarían representados Italia y $\mathrm{R}$ Unido, respectivamente. España se situaría en el quinto puesto (Gráfico 2).

Deteniéndonos a continuación en los fondos asignados en los distintos programas de iniciativa comunitaria y que anteriormente reflejamos, el total de los fondos asignados en el período 1989-1993 ascendió a 3.800 millones Ecus, elevándose la cifra a 14.275 millones de Ecus para el período 1994-1999. En este período España obtuvo el $19 \%$ del total de los créditos asignados al conjunto de las 13 iniciativas comunitarias. 


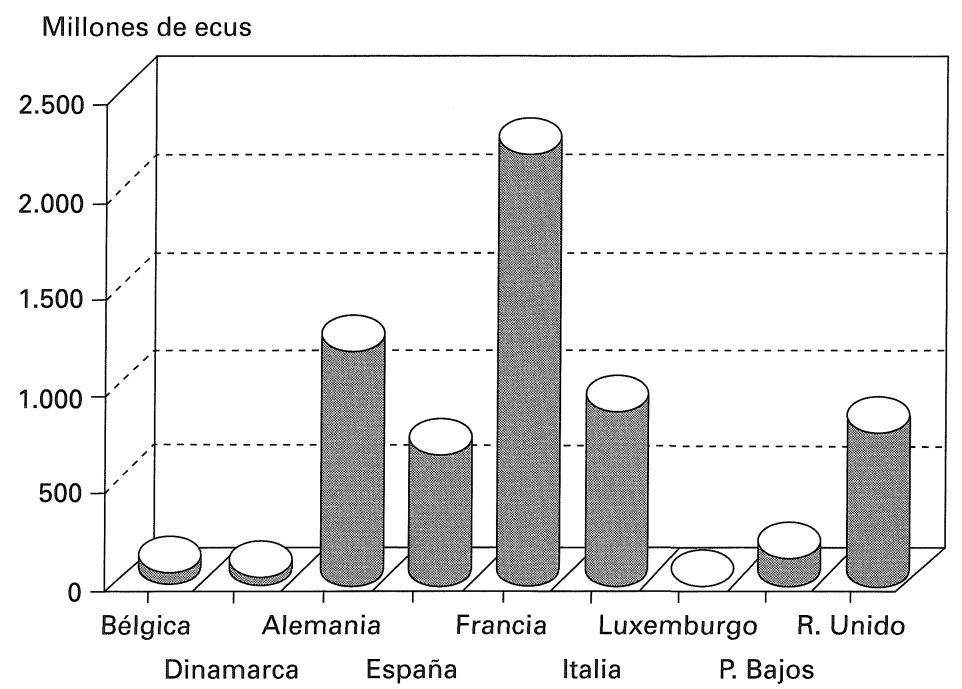

GRÁFICO 2.-Objetivo 2. 1994-1999 (mill. ecus) Diversificación zonas rurales

Informe general CEE, año 1994.

La cantidad asignada al programa LEADER ha pasado de 400 millones de Ecus (período 1989-1993) a 1.758,7 millones de Ecus (período 1994-1999), habiendo obtenido España en este último período el 23\% del total de los créditos asignados a esta iniciativa.

A pesar de la modificación al alza de las cantidades asignadas, todavía queda mucho por hacer en materia de distribución del presupuesto comunitario, dado que aunque la modificación de la Política Agrícola Común ya está permitiendo reducir las cantidades asignadas en el presupuesto comunitario al FEOGA garantía encargado de la política de sostenimiento de precios, este capítulo sigue representando un porcentaje importante en los gastos comunitarios. En relación con el total de gastos, la proporción del gasto en la PAC que se destinaba a política de precios y mercados agrarios (FEOGA-G) va decreciendo, mientras que el porcentaje del gasto en políticas estructurales (FEOGA-O, FEDER y FSE) sobre el total va en aumento (Sumpsi, 1994). En 1988, los gastos en FEOGA-G eran el 64\% del total, el 50\% en 1993, están previstos en el 46,5\% en 1998, y para 1999 supondrán el 45,6\%. En cambio los gastos en 
políticas estructurales que suponían tan sólo el 18\% en 1988, pasaron al $30,8 \%$ en 1993, han significado el 34,9\% en 1998 y en 1999 serán del 35,7\% (Comisión Europea, 1995).

La nueva visión europea del desarrollo rural: de la Conferencia de Cork a la Agenda 2000

En noviembre de 1996, ocho años después de la publicación de $E l F u$ turo del Mundo Rural, que hasta entonces había sido «el catecismo» de los planteamientos oficiales comunitarios sobre el desarrollo rural (García Bartolomé, J. M., 1994), se celebró en Cork (Irlanda) una Conferencia Europea sobre Desarrollo Rural, organizada por la DG VI de la Comisaría de Agricultura y Desarrollo Rural de la Comunidad Europea, titulada La Europa Rural-Perspectivas de Futuro, en la que se convocó a una numerosa representación de todos los medios vinculados al ámbito rural: políticos, representantes de diversas instituciones, investigadores, académicos, etc.

La Conferencia marcó una nueva etapa, decisoria en la Política Rural europea al trazar las grandes orientaciones de la misma en el horizonte $2000^{18}$. Esas orientaciones quedaron recogidas en la llamada $D e$ claración de Cork - Por un medio rural vivo, en la que se anunciaba un programa de desarrollo rural expresado en los 10 puntos siguientes:

1. Preferencia rural: El desarrollo rural sostenible debe ser una de las principales prioridades de la UE y el principio fundamental para basar toda la política rural. Para ello hay que utilizar una parte creciente de los recursos disponibles Con ello se trata de:

- Evitar la emigración rural.

- Estimular el empleo y la igualdad de oportunidades entre los sexos.

- Preservar y mejorar la calidad del medio ambiente.

- Responder a las crecientes demandas de la sociedad rural y urbana contribuyendo a su bienestar.

${ }^{18}$ La Conferencia de Cork se desarrolló en seis talleres, que correspondían a otros tantos retos para el desarrollo rural: el desarrollo rural integrado; el empleo, la igualdad de oportunidades y el espíritu de empresa en las zonas rurales; el medio ambiente y el desarrollo duradero; la calidad de vida en el medio rural; la cooperación y la transferencia de experiencias como medios de promoción del desarrollo rural; y las estrategias de desarrollo local en las zonas rurales y el enfoque ascendente. 
- Equilibrar el gasto público (inversiones, infraestructuras, educación, salud, comunicaciones) entre áreas rurales y urbanas.

2. Enfoque integrado: La política de desarrollo rural (PDR) ha de abarcar en el mismo marco jurídico y político:

- Ajuste y desarrollo agrícola.

- Diversificación económica (en especial PYMEs y servicios rurales).

- Gestión de recursos naturales.

- Reforzamiento de funciones medioambientales.

Debe aplicarse a todas las áreas rurales, con especial dedicación a la cofinanciación de las áreas más necesitadas.

3. Diversificación: Crear un marco de apoyo para la diversificación de las actividades sociales y económicas. en el que puedan sostenerse las iniciativas privadas y las comunidades de base (inversiones, asistencia técnica, formación, educación, tecnologías de la información, etc.)

4. Sostenibilidad: Conservar calidad y variedad de los paisajes rurales (recursos naturales, biodiversidad e identidad cultural), tomando conciencia de las responsabilidades globales en las actuaciones locales.

5. Subsidiariedad: Descentralizar la PDR y basarla en la asociación y la cooperación de todos los niveles (local, regional, nacional y europeo) y adoptar un enfoque «emergente» que tenga su punto de partida en la creatividad y solidaridad de las comunidades rurales. El impulso para el desarrollo rural debe hacerse a escala local y comunitaria, en un marco europeo coherente.

6. Simplificación: Evitar las excesivas complicaciones burocráticas y legislativas, sobre todo del componente agrario: no renacionalizar la PAC, pero sí hacerla más coherente simplificando los canales y limitando las reglas generales, con decisiones más subsidiarias y mayor flexibilidad.

7. Programación: La aplicacion de los Programas de Desarrollo Rural (PrDR) estará basada en procedimientos coherentes y transparentes. Cada región tendrá un único PrDR y un sólo mecanismo para el desarrollo rural sostenible.

8. Financiación: Para promover proyectos locales de desarrollo rural:

- Fomentar el uso de recursos financieros locales.

- Fomentar la ingeniería financiera en técnicas de créditos rurales para favorecer sinergias entre financiación pública y privada.

- Reducir la presión fiscal sobre las PYMEs.

- Fomentar una mayor participación del sector bancario y otros intermedios financieros.

9. Gestión: Dotar de asistencia técnica, formación y comunicaciones a los gobiernos locales y regionales y a los grupos locales, para mejorar su capacidad

$$
-600-
$$


administrativa y su eficacia. La creación de redes entre regiones y comunidades rurales de Europa, será fundamental para compartir investigaciones, información e intercambiar experiencias.

10. Evaluación e Investigación: Reforzar el seguimiento, evaluación y valoración de los beneficiarios para:

- Asegurar la transparencia de los procedimientos.

- Garantizar la correcta utilización de los fondos públicos.

- Estimular la investigación y la innovación.

- Facilitar el debate público.

Los beneficiarios deben ser consultados en el diseño y aplicación de los proyectos y sería conveniente que ellos mismos los siguieran e intervinieran en las evaluaciones.

Comparando las nuevas orientaciones con las anteriormente vigentes, vemos cómo se mantienen las líneas básicas seguidas desde 1988 y se consolidan las orientaciones iniciadas tímidamente en 1986, pero que ya en Cork quedan más definidas: la necesidad de diversificar las actividades económicas en el medio rural y no basarlo todo en la agricultura; darle un enfoque integrado, descentralizado, sostenible, con una dimensión territorial, con mayor participación de la capacidad local, con más investigación...

De la Declaración es de destacar la importancia que se concede a lo local. Y entre los elementos nuevos destacan:

- La expansión a todas las áreas rurales de las políticas de desarrollo rural, con diferencias en la cofinanciación según la necesidad de las áreas.

- La simplificación de la legislación, cuya complejidad frenaba la presentación y el desarrollo de los proyectos.

- El anuncio de un solo programa para cada región y un mecanismo para el desarrollo rural.

- El aumento de la inversión y la financiación privada en los proyectos locales de desarrollo rural.

- La subsidiariedad, descentralizando la PDR y basándola en la asociación y cooperación a nivel local, regional, nacional y europeo, aprovechando la creatividad de las comunidades rurales, con un enfoque «de abajo-arriba» y todo ello dentro de un marco europeo coherente. 
El 16 de julio de 1997, el Sr. Santer, presidente de la Comisión Europea, presentó a los parlamentarios europeos la Comunicación «Agenda 2000: por una Europa más fuerte y más amplia», en la que en un documento de 1.300 páginas se abordaban tres temas principales: las perspectivas generales de desarrollo de la UE y de sus distintas políticas de cara al siglo que viene, los problemas horizontales relacionados con la ampliación, y el futuro marco financiero en el que se tendrá en cuenta que más allá del año 2000 la UE contará con nuevos países miembros ${ }^{19}$.

Con la mirada puesta en el mundo rural, se puede decir que la Comisión afrontó tres grandes retos:

- Ampliación de la Unión a 10 países de Europa Central y Oriental, así como a Chipre.

- Remodelar la política estructural comunitaria para mantener las ayudas que se conceden para el desarrollo regional y social de los 15 países miembros y seleccionar con mayor precisión los objetivos, que también serán aplicados a los futuros estados miembros de forma progresiva.

- Ampliar la reforma de la PAC para garantizar la competitividad de la agricultura europea y la viabilidad de la economía rural, considerando las exigencias de los consumidores, la evolución del mercado mundial y la ampliación de la Unión a nuevos países.

Sin entrar en cuestiones profundas referentes al primero de los retos mencionados, sí conviene decir que los problemas rurales de los países candidatos constituirán una dimensión importante de la intervención comunitaria en esos países a nivel financiero ${ }^{20}$.

${ }^{19} \mathrm{El}$ Sr. Santer indicaba en esa fecha la posibilidad de nuevas negociaciones de adhesión con cinco países del Este, además de Chipre: Hungría, Polonia, Estonia, la República Checa y Eslovenia. Actualmente ya está en curso. Las puertas están abiertas para Bulgaria, Letonia, Lituania y Eslovaquia, que pasarán por un acuerdo de asociación previa a la adhesión. Pero la ampliación de las fronteras de la UE, puede llegar a Ucrania, Bielorrusia y Moldavia.

${ }^{20}$ La Comisión propuso asignar a los nuevos Estados miembros 45.000 millones de Ecus procedente de los Fondos Estructurales y del Fondo de Cohesión. Desde el año 2000, y procedentes de esa asignación, gozarían de una ayuda previa a la adhesión de 1.000 millones de Ecus al año. 
El desarrollo rural después de la Agenda 2000: una Política con entidad propia

Con objeto de llevar a cabo las reformas políticas propuestas en la Agenda 2000, el 18 marzo de 1998 la Comisión presentó una serie de propuestas legislativas conteniendo los textos jurídicos que proporcionarán la base para las decisiones que deberán adoptarse. En las mencionadas propuestas, y en lo que se refiere al mundo rural, están presentes las orientaciones y recomendaciones que se dieron en la Conferencia de Cork.

A) Nuevos Reglamentos sobre los Fondos estructurales y el Fondo de Cohesión para el periodo 2000-2006). - Con la remodelación de la política estructural, se quiere conseguir una mayor eficacia de las acciones estructurales comunitarias, para lo cual se ha propuesto reducir el número de objetivos a tres, reducción que no supone un debilitamiento del esfuerzo que se viene haciendo en materia de cohesión económica y social. En temas financieros se mantiene la solidaridad financiera en su nivel actual del $0,46 \%$ del PNB, lo que supone para el período 2000-2006 una dotación de 275.000 millones de Ecus, de los cuales se destinarán a los Fondos estructurales propiamente dichos 210.000 millones de Ecus (a precios de 1997) para los 15 países miembros en el citado período.

Esta remodelación, que ha obligado a una nueva redacción de los Reglamentos sobre los fondos estructurales y del fondo de cohesión para el período 2000-2006 (Commission Européenne, 1998), tiene como contenido básico los siguientes objetivos prioritarios:

OBJETIVO 1: Promover el desarrollo y el ajuste estructural de las regiones atrasadas.

(El título de este objetivo no ha cambiado, pero la propuesta de la Comisión preve que las zonas hoy elegibles con el Objetivo 6 así como las regiones ultraperiféricas se integren en el Objetivo I en el período 2000-2006).

La Comisión deberá elaborar en el futuro la lista de regiones subvencionables en virtud de este objetivo sobre la base de una aplicación rigurosa del criterio del PIB inferior al 75\% de la media de la Unión.

En términos de concentración financiera el Obj. 1 debería contar con aproximadamente dos tercios del total de las ayudas de los Fondos estructurales. La

$$
-603-
$$


cuantía de la ayuda será proporcional al volumen de población, a la prosperidad nacional y a la diferencia entre la prosperidad regional y el promedio comunitario. Habrá ayudas suplementarias para regiones con grave subempleo.

Fondos que intervienen: FEDER FSE, FEOGA-O e IFOP.

OBJETIVO 2: Sostener la reconversión económica y social de las zonas con dificultades estructurales

Este nuevo Objetivo, centrado en la reconversión económica y social, reúne los Objetivos 2 y 5 b del periodo actual y se amplía a otras zonas (zonas urbanas en dificultad, zonas en crisis dependientes de la pesca y zonas en reconversión muy dependientes de los servicios) enfrentadas al mismo problema de escasa diversificación económica).

La lista de zonas subvencionables será elaborada por la Comisión en estrecha colaboración con los Estados miembros y teniendo en cuenta sus prioridades.

La Comisión preve que la población abarcada por este Objetivo se limite al 18\% de la población total de la Unión. Pero también propone que, en cada Estado miembro, esta población (incluida la de las regiones que se acogían al Objetivo 1 y que satisfacen los criterios para ser subvencionables en virtud de este nuevo objetivo) no sea reducida en más de un tercio con respecto a la población abarcada actualmente por los Objetivos 2 y 5 b (que incluyen áreas rurales).

Las zonas rurales en declive de este Objetivo 2 deberán cumplir, en lo que concierne como mínimo al $50 \%$ de la población afectada, dos de los cuatro criterios siguientes:

- Una densidad de población inferior a 100 habitantes por $\mathrm{km}^{2}$ o un índice de desempleo en el sector agrario igual o superior al doble de la media de la Unión, y

- Un índice medio de desempleo superior a la media de la Unión o un descenso de población.

Las zonas que actualmente son beneficiarias de los objetivos 2 y $5 \mathrm{~b}$, que dejen de serlo de acuerdo con los futuros criterios de selección, seguirán beneficiándose de una ayuda limitada durante un período transitorio.

Fondos que intervienen: FEDER, FSE, FEOGA-G e IFOP.

OBJETIVO 3: Sostener la adaptación y la modernización de las políticas y sistemas de educación, de formación y de empleo.

(Este muevo Objetivo reúne los anteriores Objetivos 3 y 4. Interviene fuera de las zonas elegibles por los nuevos Objetivos 1 y 2 , pero sirve igualmente de marco de referencia para el conjunto de desarrollo de recursos humanos en cada Estado miembro). 
Este objetivo fomentará las actividades en los cuatro ámbitos siguientes:

- Acompañamiento de transformaciones económicas y sociales.

- Sistemas de educación y formación durante toda la vida.

- Política activa de lucha contra el desempleo.

- Promoción de la inserción social y la igualdad de oportunidades para mujeres y hombres.

Fondos que intervienen: FSE.

Siguiendo la información anterior, en los Objetivos 1 y 2 toman parte el FEDER y el FEOGA. Este segundo Fondo en su sección Orientación actuará en zonas Objetivo 1 y en su sección Garantía en las zonas Objetivo 2. A pesar de que el FEOGA-Garantía no es un fondo estructural, financiará acciones estructurales de desarrollo rural dentro de las distintas OCM (Calatrava, A. y Melero, A. 1997) y también medidas de acompañamiento de la PAC y del desarrollo rural, fuera de las zonas elegibles en los Objetivos 1 y 2, como consta tanto en la propuesta del Reglamento General de los Fondos estructurales (arts. 28 y 29) como en la propuesta de Reglamento del Consejo sobre la ayuda al desarrollo rural a cargo del FEOGA (art. 45). En el objetivo 3, interviene el FSE.

En lo referente a las iniciativas comunitarias, la Comisión ha propuesto reducir el número de las 13 que están vigentes hasta 1999 a únicamente 3, que hacen referencia a los siguientes ámbitos:

- La cooperación transfronteriza, transnacional e interregional, orientada tanto a fomentar el desarrollo económico de las regiones como a promover la ordenación económica y equilibrada del territorio.

- Los recursos humanos, para lograr nuevas prácticas de lucha contra las discriminaciones y desigualdades de todo tipo en el acceso al mercado laboral.

- El desarrollo rural.

A las 3 iniciativas se asignará el 5\% de los recursos de los Fondos estructurales (anteriormente era el 9\%), es decir un presupuesto que ronda los 10.500 millones de Ecus.

B) Nuevos Reglamentos agrarios.-De las diferentes propuestas de Reglamentos agrarios, destacamos aquí el nuevo Reglamento que establece las medidas de desarrollo rural financiadas por las dos secciones 
del FEOGA: La sección Orientación en las zonas del Objetivo 1, y la sección Garantía en las demás zonas.

En materia agrícola, las propuestas de la Comisión inciden en la profundización y extensión de la reforma de 1992, sustituyendo por más ayudas directas las medidas de sostenimiento de precios, y acompañando estas actuaciones con una política rural coherente.

Es evidente que el desarrollo rural no puede considerarse un anexo de la PAC o un suplemento de la misma; es uno de sus pilares esenciales y como tal necesita una política rural coherente que articule mejor el desarrollo rural y la política de mercados y que valorice todas las facetas del desarrollo rural, fomentando la participación de los agentes locales y la creación de empleos.

\section{La política de desarrollo rural en la Europa del siglo XXI}

Con los nuevos Reglamentos va a ser superado el dualismo que actualmente persiste entre política de estructuras agrícolas y política de desarrollo rural.

La nueva política rural, por un lado, ofrecerá un adecuado apoyo al proceso de modernización de las estructuras agrarias de producción, por otro, responderá a las exigencias de las poblaciones rurales y a las necesidades de un medio ambiente frágil pero también rico en recursos que hay que desarrollar.

En el momento que se redactan estas páginas, ya se ha aprobado el Reglamento sobre ayuda al desarrollo rural a cargo del FEOGA que será aplicable a las ayudas comunitarias a partir del 1 de enero de 2000. El nuevo Reglamento tiene como fin asistir y completar las reformas propuestas en la política de precios y mercados agrarios y supone una simplificación sustancial de los distintos instrumentos de política rural actualmente en vigor ${ }^{21}$. Define como objetivo prioritario la modernización y diversificación del sector agrícola y silvícola y establece

21 El Nuevo Reglamento (Reglamento CE núm. 1257/1999 del Consejo, de 17 de mayo de 1999 y publicado en el DOCE del 26 de junio de 1999) reorganiza en un único texto los nueve Reglamentos que actualmente hacen referencia al desarrollo rural: El Reglamento denominado «Fondos estructurales» referente al FEOGA, los cuatro Reglamentos «Objetivo 5a», los tres Reglamentos que hacen referencia a necesidades de acompañamiento, y el Reglamento relativo a la ayuda estructural en favor de la silvicultura. 
los objetivos de intervención en materia de mantenimiento de actividades económicas vinculadas en particular a mantenimiento y creación de empleos, mejora de las condiciones de vida y de trabajo, protección del medio ambiente, etc.

Las medidas de desarrollo rural subvencionables en virtud del nuevo Reglamento pertenecen a dos grupos:

- las medidas complementarias de la reforma de 1992; jubilación anticipada, medidas agroambientales y repoblación forestal, así como el régimen aplicable a las zonas desfavorecidas;

- las medidas de modernización y de diversificación de las explotaciones agrícolas: inversiones en explotaciones agrícolas, instalación de jóvenes agricultores, formación, apoyo a las inversiones en instalaciones de transformación y comercialización, ayuda complementaria a la silvicultura y actividades que tengan como objetivo la reconversión y mejora de las actividades ligadas a la agricultura.

La aplicación de estas medidas supone importantes mejoras con respecto a las de la situación actual, muchas de ellas con repercusiones económicas directas que supondrán importantes incrementos en las ayudas que venían recibiendo los agricultores. En su aplicación destacan entre las de mayor importancia económica para España (UAGN 1999):

Mejora y modernización de explotaciones: Se incrementan los niveles máximos de ayuda en una media del $25 \%$ sobre la percibida anteriormente.

Instalación de jóvenes agricultores: Se aumenta el 66,7\% el importe de las ayudas para los menores de 40 años que se establezcan por primera vez como titulares de una explotación agraria económicamente viable y que respete unas normas mínimas medioambientales. En la actualidad el máximo que se podía percibir eran 5 millones de pesetas, y se ha aumentado a 8,3 millones de pesetas.

Indemnizaciones compensatorias: Aplicables al cese de la actividad agraria y a las zonas desfavorecidas y con limitaciones medioambientales específicas. En España supondrá que el 90\% del territorio nacional tendrá derecho a esas indemnizaciones y permitirá recibir ayudas a 17.000 agricultores que hasta ahora no tenían compensaciones. 
Forestación de tierras agrarias: Para compensar las diferencias de ingresos por forestación de tierras agrarias se incrementará en un 20\% el importe máximo de las primas actuales.

Ampliación a todo el territorio nacional: Desaparece la limitación de ayudas a las regiones Objetivo 1 y zonas 5b. En España, hasta ahora, quedaba fuera el $26,3 \%$ del territorio.

Desde enero del año 2000, en todas las regiones de la UE (incluso en las regiones del Objetivo 1 en los que la programación se basa en políticas estructurales) habrá diversas medidas cofinanciadas únicamente por el FEOGA-Garantía: las medidas que acompañaban a la reforma de la PAC de 1992; las medidas conocidas como medidas «5a»; y las medidas de adaptación y de desarrollo rural que en la actualidad y hasta 1999 están limitadas al objetivo 1 y a las zonas rurales objetivo 5b. Todas estas medidas tendrán una aplicación horizontal en las zonas no pertenecientes a Objetivos regionalizados y se organizarán dentro de un mismo programa de ámbito nacional, aunque también por iniciativa de los Estados, podrá descentralizarse en el nivel territorial que sea más conveniente (INFO-LEADER 1998).

A partir del año 2000, la política de desarrollo rural ya no será sólo, como ahora, un elemento de la política de cohesión económica y social, sino que también será un instrumento de acompañamiento de las reformas propuestas en el ámbito de la PAC sobre precios y mercados y todo ello para la totalidad de las regiones rurales de Europa. El mensaje que deja traslucir la Comisión es que el objetivo estratégico de la política de desarrollo rural es doble (Silva, J. M., 1998):

- Garantizar en el sector agrícola y en el medio rural un futuro de larga duración constituido por oportunidades de empleo, de calidad de vida y de medio ambiente.

- Responder a las exigencias de adaptación estructural del sector y al desarrollo socioeconómico de las zonas rurales.

El objetivo de la Política Rural Integrada Comunitaria (PRIC) será alcanzar un equilibrio más sostenible entre la actividad agrícola, otras formas de desarrollo rural y la conservación de los recursos naturales (García Azcárate, T., 1996). Un objetivo realmente ambicioso y cuyo logro no está exento de dificultades (Etxezarreta, M., 1988), ya que si una 
de las finalidades del Desarrollo Rural Integrado (DRI) es la creación de empleos, son necesarias no sólo grandes dosis de entusiasmo por parte de los emprendedores rurales y de los agentes de desarrollo local sino también un enorme esfuerzo en formación y mucha capacidad para no perder los ánimos si los resultados apetecidos no se producen en el corto plazo. El desempleo rural es de enorme extensión, sobre todo entre la población agrícola, y no es fácil absorber a la población parada en nuevos empleos (Etxerreta, M., 1994).

Las nuevas orientaciones de la Política Estructural de la UE suponen la aceptación de algo evidente: el modelo de crecimieno concentrado y desarrollado «desde arriba» se ha ido desdibujando en los últimos años, dando paso a un nuevo paradigma de desarrollo autocentrado y difuso, basado en la utilización productiva de los recursos locales (Furió Blasco, 1994). Las estrategias de desarrollo «desde abajo» están sustentadas por factores no solamente económicos sino también sociales, culturales y territoriales que constituyen las potencialidades de desarrollo endógeno de un área (Vázquez Barquero, 1986).

A partir del año 2000 se abren nuevas perspectivas para las zonas rurales de la UE. La nueva política de desarrollo rural que va a aplicarse en el próximo siglo tendrá en consideración todas las zonas rurales y todas serán beneficiarias potenciales de recursos ya que es de esperar que, vistos los resultados positivos logrados por los grupos de acción local beneficiarios de las ayudas de las iniciativas LEADER I y LEADER II, se sigan presentando Proyectos de desarrollo rural que utilicen recursos endógenos y sean capaces de generar empleos y mantenerlos.

\section{BIBLIOGRAFÍA}

BANDARRA, N (1992): «Fondos estructurales comunitarios y cohesión después de Maastricht». Revista de Estudios Agro-sociales, núm. 159, MAPA.

Berger, A. y Rouzier, J (1995): «L'espace rural, élargissement conceptuel et orientation méthodologique». Économie Rurale, núm. 229, pp.3-10.

Blanc, M. (1997): «La ruralité: diversité des approches», Économie Rurale, núm. 242, pp. $5-12$

Blunden, J. R.; Pryce, W. T. R. y Dreyer, P. (1998): «The classification of rural areas in the european context: an exploration of a typology using neural network applications», $R e$ gional Studies. Vol. 32.2. pp. 149-160.

Calatrava, A. (1994): «El espacio rural como campo de experimentación para un nuevo modelo económico». II Seminario Iberoamericano. Inter-acción, Navapalos (Soria).

Calatrava, A. y Melero, A. (1995): «La educación ambiental en el desarrollo sostenible». 
Primer Curso Internacional intensivo sobre Desarrollo Sostenible. El Burgo de Osma (Soria).

- (1997): «Las Organizaciones Españolas de Productores de Frutas y Hortalizas ante los retos actuales del mercado europeo, en Mundialización, Innovación, Región, Arco Mediterráneo, pp. 203-210, AECR y Universidad Politécnica de Valencia, Valencia.

Canto Fresno, C. Del y Casablanca, F. DE (1996): Innovación y Desarrollo en Áreas Rurales, Ministerio de Agricultura Pesca y Alimentación, Madrid.

COM (87): «Situacion y evolución socioeconómica de las regiones comunitarias». Doc. (COM (87) 250 final y Bol. CE 5-1987 punto 2.1.127).

Comisión de las Comunidades Europeas (1986): Acta Unica Europea. Boletín de las Comunidades. Luxemburgo.

- (1988): El futuro del mundo rural, Comunicación de la Comisión. 29 de julio COM(88) 501 final, Boletín de las Comunidades Europeas. Suplemento 4/88.

- (1989): Vademecum sobre la reforma de los fondos estructurales. Luxemburgo.

Comisión EuropeA (1994): Guía de iniciativas comunitarias 1994-1999, OPOCE, Luxemburgo.

- (1995): Informe General sobre la actividad de la Comisión Europea. OPOCE. Luxemburgo.

- (1996): Fondos estructurales y Fondo de Cohesión. Textos reglamentarios y comentarios. OPOCE, Luxemburgo.

(1997): Ayudas y préstamos de la UE, Ed. EUR-OP.

COMMISSION EUROPÉENNE (1995): Livre Vert sur l'Innovation, Décembre 1995.

— (1998): Propositions de Réglements des Fonds Estructurels 2000-2006. Analyse comparé.

CoRdero, G. (1993): «Política Regional y desarrollo local», en Desarrollo local y medio ambiente en zonas desfavorecidas, pp. 177-185, Ed. MOPT. Madrid.

Etxezarreta, M. (Comp.) (1988): Desarrollo rural integrado, MAPA. Serie Estudios, núm. 50. Madrid.

- (1994): «Trabajo y Agricultura: cambios en el sistema de trabajo en una agricultura en transformación», Revista Agricultura y Sociedad, núm. 72, MAPA, Madrid.

European Commission DG VI (1997): CAP 2000. Working document. Situation and Outlook: Rural Developments, Directorate Gral. for Agriculture (DG VI), July 1997.

EUROSTAT (1993): Revenu Agricole, OPOCE, Luxemburgo.

Furió Blasco, E. (1994): «El desarrollo económico endógeno y local: reflexiones sobre su enfoque interpretativo», Estudios Regionales, núm. 40, pp. 97-112.

GARCÍA AzCÁRATE, T. (1996): «La política agraria común a debate», Revista Española de Economía Agraria, núm. 176-177. pp. 252.

García BARTolomé, J. M. (1994): Una visión del desarrollo rural a través de las publicaciones del Ministerio de Agricultura, Pesca y Alimentación (MAPA) (19601995), Secretaría Gral. del MAPA. Madrid.

García SANZ, B. (1996): La sociedad rural ante el siglo XXI, MAPA, Serie Estudios, núm. 125. Madrid.

INFO-LEADER (1998): Un nuevo marco para el desarrollo rural en Europa, núm. 59, marzo 98. Suplemento. Comisión Europea. Bruselas.

LÁZARO, L. (1995): «La política regional y la cohesión económica y social en la Comunidad Europea». Primeras Jornadas de desarrollo estratégico provincial. pp. 127-158, Ed. Excma. Diputación Provincial de Cuenca.

OCDE (1992): Entreprises et Emplois dans le Monde Rural París, 1992.

(1994): Creating rural indicators. París, 1994.

SILVA, J. M. (1998): «El desarrollo rural en la Europa del próximo siglo. La Agenda 2000», ponencia presentada en el Congreso Internacional de Desarrollo Rural: El Desarrollo 
agrario y el Desarrollo rural Los agricultores: Nuevos actores del desarrollo, Valladolid, 3-5 junio.

Sumpsi Viñas, J. M. ${ }^{a}$ (1994): «La Política Agraria y el Futuro del Mundo Rural», Revista de Estudios Agrosociales, núm. 169, julio-septiembre 1994, pp. 150-173.

UAGN (1999): «El Reglamento de Desarrollo Rural», Boletín Informativo de la Unión de Agricultores y Ganaderos de Navarra, núm. 240, agosto 1999, pp. 24-25.

VALCÁRCEL-RESALT, G. (1993): «Planes estratégicos de desarrollo local», en Desarrollo Local Turismo y Medio Ambiente, pp. 21-55, Ed. Excma. Diputación Provincial de Cuenca.

VÁZQUEZ BARQUERO, A. (1986): «El cambio del modelo de desarrollo regional y los nuevos procesos de difusión en España», Estudios Territoriales, núm. 20. pp. 87-110. 